\title{
Biliary Tract Carcinoma
}

National Cancer Institute

\section{Source}

National Cancer Institute. Biliary Tract Carcinoma. NCI Thesaurus. Code C156781.

A carcinoma arising from the intrahepatic bile ducts, hepatic ducts, common bile duct, cystic duct, or gallbladder. 